\title{
Prevalence of Anxiety and Depression amongst Patients presenting with Non-inflammatory Vaginal Discharge.
}

\author{
Sayed Shah Khalid', Shafqat Huma², Usman Amin Hotiana ${ }^{3}$, Muhammad Tariq ${ }^{4}$, Muhammad Nadeem ${ }^{5}$
}

\section{ABSTRACT}

\begin{abstract}
Background: Abnormal vaginal discharge/leucorrhea amongst women of reproductive age group(15-49) is a frequently encountered complaint especially across South-east Asia. It is commonly associated with tremendous stress, anxiety and depression. The current study was planned to determine the prevalence of anxiety and depression amongst such patients.
\end{abstract}

Objective: To stydy, the prevalence of anxiety and depression as a cause of vaginal discharge in young women.

Design: Descriptive, cross-sectional study

Place and duration of study: Combined Military Hospital,Kohat, for a period of 3 months from June 2015 till September 2015.

Material and Methods: 62 consecutive patients presenting with non-infectious, chronic, leucorrhea were inducted from Gynecology Outpatient Department, Combined Military Hospital. As per the inclusion criteria for this study, any female aged 1845 years presenting to Gynaecology outpatient services with chronic non-infective vaginal discharge was eligible for the study. Patients with comorbid mental retardation, psychotic disorders, or organic brain syndromes were excluded.

Their basic demographic details were recorded through a sociodemographic proforma and then Hospital Anxiety and Depression Scale was administered orally in the native language to the participants.

The data was entered in the computer and analysed using SPSS 22.0. and results interpreted accordingly.

Results: $26(42.6 \%)$ of cases were normal, $10(16.4 \%)$ had borderline anxiety symptoms, and $25(41 \%)$ cases had abnormal/severe anxiety; regarding depression, it was found that $34(55.7 \%)$ were normal cases, $11(18 \%)$ borderline and $16(26.2 \%)$ were abnormal/severe according to Hospital and anxiety scale.

Conclusion: Clinically significant anxiety and depression are frequently reported in patients presenting with chronic leucorrhea. Appropriate and timely management of these could be better health related ou tcomes.

Keywords: Leukorrhea, vaginal discharge, anxiety, depression

This article may be cited as: Khalid SS, Huma S, Hotiana AU, Tariq M, Nadeem M.

Prevalence of Anxiety and Depression amongst Patients presenting with Non-inflammatory Vaginal Discharge. J Saidu Med Coll Swat 2020:10(1):65-69

\section{INTRODUCTION}

Abnormal vaginal discharge is frequently reported and about a quarter of women experience it. ${ }^{1,2,3}$ Vaginal discharge is deemed as an indication of reproductive tract infections ${ }^{4}$ and it is quite prevalent across the subcontinent. ${ }^{5}$ Conversely, recent literature reveals that such infections are much less reported amongst women who have abnormal discharge from the vagina. ${ }^{6-8}$

Secretions from the genitalia are deemed as a pure form of "Dhat", the loss of which could cause progressive weakness or ultimately even death. ${ }^{9,10,11}$ Most patients with these complaints often experience symptoms of anxiety, depression, distress and constant worry about it. Most South Asian women with vaginal discharge also experience different other bodily symptoms like lightheadedness, backache, and weakness. ${ }^{13-}$ 20

In an ethnographic study, Trollope-Kumar ${ }^{10}$

\footnotetext{
1.Psychiatry Deptt, CMH, Sarghoda

2.Psychiatry \& Behavioral Sciences, University of Lahore

3.Psychiatry Deptt, King Edward Medical University, Lahore

4.Psychiatry Deptt, Sahara Medical College \& Hospital, Narowal

5.Surgery Deptt, Saidu Teaching Hospital, Swat
}

Correspondence Address:

Lt. Col. Syed Shah Khalid

HOD Psychiatry CMH Sargodha Cantt noticed the worries of women regarding the loss of genital secretions often reporting discharge of "safed panni"(white water), "dhatu", or "swed pradhar". Loss of these secretions was found to be linked with vague presentations in the form of burning hands and feet, lightheadedness, backache, and progressive weakness in the body. These women associated the symptoms to loss of an essential fluid from the body. On thorough assessment, these women hardly had any evidence of infection and the quantity of discharge did not seem to be more than the normal physiological discharge.

It is noteworthy that not only the women but few local health care professionals (e.g., dai) were also perturbed about "safed panni" owing to the common belief that "100 drops of blood was needed to make a drop of safed panni," a belief which is quite same as seen in male patients with Dhat syndrome. ${ }^{9,11}$ Also, these women related the whitish vaginal discharge to variety of factors such as tubectomy and improper diet. ${ }^{10}$

Researchers who have assessed women with major psychiatric disorder, also described leukorrhea as a somatic means of expression of depression in South Asia ${ }^{16,17,18,19}$. In few of these studies, patients related their symptom of vaginal discharge to stress and emotional upsets, excess 
body heat, infection, use of hot and spicy foods, and effect of hot weather. . $^{17,18,19}$

Thus, keeping this background in mind, and the fact that there is paucity of research in this area in our country, we decided to conduct this study to find out the prevalence of anxiety and depression in patients presenting with non-infective leucorrhea, in a tertiary care setting.

\section{MATERIALAND METHODS}

This study was carried out for a 3 months period from 15thJune 2015 to 15th September 2015 at Gynaecology Outpatient Department, Combined Military Hospital, Kohat. The research was commenced after taking formal permission from Hospital Research Ethical Committee and written informed consent was obtained from all concerned patients. They were explicitly explained the aims and objectives of the research and offered reassurance that their confidentiality shall be maintained throughout the study.

The sample size was calculated, keeping power $(1-\beta)=0.80$, confidence interval $=95 \%$, and margin of error $(\alpha)=5 \%$, which was 61 patients. These patients were consecutively recruited from the Gynaecology Outpatient Department of Combined Military Hospital,Kohat. As per the inclusion criteria for this study, any female aged 18-45 years presenting to Gynaecology outpatient services with complaints of excessive vaginal secretions for more than 6 months(thus defining chronic lecorrhea) with the following associated features ${ }^{22}$ :

Abnormal colour; brown, green, tinged with blood

Abnormal consistency; thicker, more watery

Loss of cyclical pattern

Obnoxious smell

Linked with other symptoms; itch, soreness, painful sexual intercourse. were eligible for the study. Only those patients whose gynecological treatment records and investigation reports for common bacterial, parasitic, and fungal infection ruled out any infective cause for the complaint of vaginal discharge were considered further for this study. This assessment was performed by a senior gynaecologist, with a proper specialist qualification FCPS/FRCOG or equivalent with at least 5 years of extensive experience in the field.

Patients with comorbid mental retardation, psychotic disorders, or organic brain syndromes were excluded, by proper assessment performed by a consultant psychiatrist, with a proper specialist qualification(FCPS/MRCPsych or other equivalent qualification) with at least 5 years of extensive field experience. Relevant questions were asked to rule out mental retardation/intellectual disability i.e history of developmental delays, learning difficulties and poor adaptive skills; major psychosis/schizophrenia- exploration of symptoms such as odd behavior, self talking and self-smiling, hallucinatory behavior, paranoid ideation; and organic brain syndromes(i.e Delirium/dementia)-exploring for any evidence of cognitive decline as in disorientation in time, place and person, recent memory problems etc.

Their basic demographic details were recorded through a sociodemographic proforma and then Hospital Anxiety and Depression Scale ${ }^{23}$ was administered orally in the native language to the participants. The data was entered in the computer and analysed using SPSS 22.0. For the continuous variables, Mean \pm S.D was calculated. For the categorical variables, frequencies and percentages were presented

\section{RESULTS}

The mean age of the participants was $30.02 \pm$ 3.528 with an age range of $22-36$ years. (Table- 1 )

Table 1. Age distribution of the participants $(n=62)$

\begin{tabular}{|l|c|c|c|c|c|}
\hline & $\mathrm{N}$ & Minimum & Maximum & Mean & Std. Deviation \\
\hline age of patients & 61 & 22 & 36 & 30.02 & 3.528 \\
\hline
\end{tabular}


Table 2. Educational status of the participants $(n=61)$

\begin{tabular}{|l|r|r|}
\hline Educational status & Frequency & Percent \\
\hline No formal education & 8 & 13.1 \\
educated till class 5 & 9 & 14.8 \\
class 8 & 9 & 14.8 \\
intermediate & 18 & 29.5 \\
bachelors & 9 & 14.8 \\
Masters and above & 8 & 13.1 \\
Total & 61 & 100.0 \\
\hline
\end{tabular}

Regarding the educational status of the patients, $8(13.1 \%)$ had no formal education, $9(14.8 \%)$ were educated till class $5^{\text {th }}, 9(14.8 \%)$ were educated till class $8^{\text {th }}, 18(29.5 \%)$ had acquired education till intermediate/Fsc, $9(14.8 \%)$ till Bachelors, and 8(13.1\%) till Masters and above. (Table 2)

When Hospital and anxiety scale was administered, it was identified that $26(42.6 \%)$ of cases were normal, $10(16.4 \%)$ had borderline anxiety symptoms, and $25(41 \%)$ cases had abnormal/severe anxiety, whereas regarding different severities of depression, it was found that $34(55.7 \%)$ were normal cases, $11(18 \%)$ borderline and 16 (26.2\%) were abnormal/severe according to Hospital and anxiety scale, as depicted in the figure below:

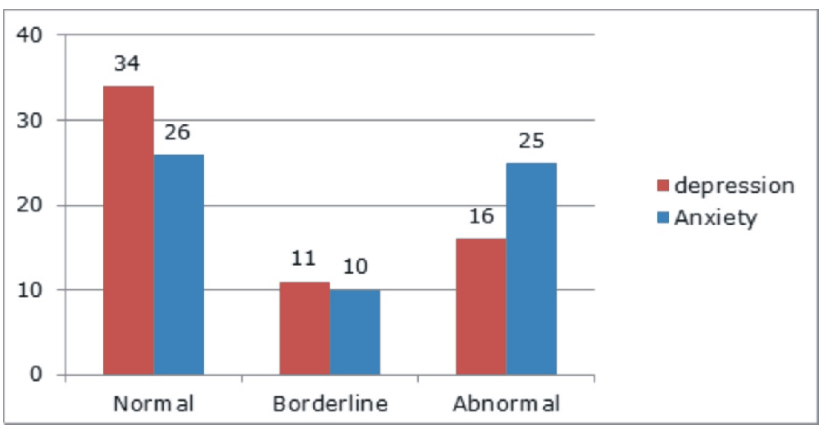

Figure 1: showing the different grades of anxiety and depression amongst patients presenting with chronic non-infective vaginal discharge $(n=61)$

\section{DISCUSSION}

The major psychiatric illnesses such as depression and anxiety disorders, are counted as the most commonly reported health problems in the community. There is an abundant literature from the West depicting a strong relationship between symptoms including abnormal vaginal disorder and psychiatric illnesses. ${ }^{21}$

A research conducted in India found very high rates of depression for patients who were attending a gynaecological clinic versus a general medical clinic.Presumably, the presentation of abnormal vaginal secretions in this part of the world is a common manifestion of stress which should not be overlooked. The reproductive health care programs must incorporate psychosocial interventions as a necessity to address these problems. $^{21}$

This study was conducted in Kohat to determine the prevalence of anxiety and depression in patients presenting to Gynecology OPD with noninfective chronic leucorrhea. The results revealed that $26.2 \%$ cases had marked depression while $18 \%$ were borderline abnormal, and regarding anxiety, $41 \%$ reported significant anxiety while $16.4 \%$ cases were borderline. These findings are comparable with other studies conducted locally and internationally which are worth discussing here.

Previous studies too have identified a higher prevalence of common psychiatric disorders in patients with vaginal discharge and have also reported vaginal discharge as a symptom in patients with common mental disorders. ${ }^{16-19}$ Hence, all women with noninfective vaginal discharge must be evaluated for mental health problems. 
Researchers who In few of these studies, patients related their symptom of vaginal discharge to stress and emotional factors, excess heat in the body, infection, use of hot and spicy foods, and effect of hot weather. ${ }^{17,18,19}$

Research particularly from South-East Asia highlights a strong link between abnormal vaginal secretions and psychosocial distress, as also emphasized previously. It is worthwhile now to discuss this study which compared 120 women attending a gynaecology clinic versus 55 women with no physical health issues. This was a case control study which found that $50 \%$ of the former group had major mental health problems as compared to only $26 \%$ for the control group. ${ }^{21} \mathrm{We}$ can clearly relate this finding to our research and see that this prevalence is much higher, which could probably be due to a large sample size of the aforementioned population concerned.

Yet another research conducted in Mumbai, India, found that half of the women experienced at least a single gynecological problem while about $25 \%$ had a mental health problem. The co-existence was more common for the younger age groups. In another study from rural Maharashta, white vaginal secretion was the most common reported issue. $^{22}$

A few common life events like psychological trauma in the form of physical and psychological violence, with almost no social support and poor spousal relationships may increase the risk of depressive illness. Weakness is very frequently reported problem amongst such women who also commonly experience symptoms of psychosocial distress and depression. ${ }^{23-25}$

So we can clearly establish a very strong link between depression and anxiety amongst young women particularly and abnormal vaginal discharge i.e leucorrhea. It calls for attention of the health care providers to address this issue, devise appropriate plan for its early identification and effective management, for better health related quality of life of the patients concerned, There are a few limitations to this study. For instance, the sample size being small limits the generalisability of the research findings to a larger population. Also, another methodological shortcoming is the absence of a control group for comparison. We should have also probed into other associated social factors leading to anxiety and depression, which was not done in this study.

\section{CONCLUSION}

The study revealed high rates of anxiety and depression amongst young women presenting to the Gynaecology Outpatient Department, with complaints of chronic non-infective vaginal discharge. Thus this issue should be properly addressed, leading to early identification and management of these serious mental health problems, thus accounting for better quality of life of patients concerned.

\section{REFERENCES}

1. Venugopal S, Gopalan K, Devi A, Kavitha A. Epidemiology and clinico-investigative study of organisms causing vaginal discharge. Indian J Sex Transm Dis AIDS. 2017;38:69-75.

2. Meena V, Bansal CL. Study to Evaluate Targeted Management and Syndromic Management in Women Presenting with Abnormal Vaginal Discharge. . J Obstet Gynaecol India. 2016 ;66:534-40.

3. Rajalakshmi R, Kalaivani S. Prevalence of asymptomatic infections in sexually transmitted diseases attendees diagnosed with bacterial vaginosis, vaginal candidiasis, and trichomoniasis. Indian J Sex Transm DisAIDS. 2016;37:139-142

4. Van Schalkwyk J, Yudin MH; INFECTIOUS DISEASE COMMITTEE. Vulvovaginitis: screening for and management of trichomoniasis, vulvovaginal candidiasis, and bacterial vaginosis. J Obstet Gynaecol Can. 2015;37:266-274

5. Diadhiou M, Ba Diallo A, Barry MS, Alavo SC, Mall I, Gassama O, et al. Prevalence and Risk Factors of Lower Reproductive Tract Infections in Symptomatic Women in Dakar, Senegal. Infect Dis (Auckl). 2019;12:11-78

6. Verwijs MC, Agaba SK, Sumanyi JC, Umulisa MM, Mwambarangwe L, Musengamana V, et al. Targeted point-of-care testing compared with syndromic management of urogenital infections in women (WISH): a cross-sectional screening and diagnostic accuracy study. Lancet Infect Dis. 2019;19:658-669.

7. Zemouri C, Wi TE, Kiarie J, Seuc A, Mogasale V, Latif A, et al. The Performance of the Vaginal Discharge Syndromic Management in Treating Vaginal and Cervical Infection: A Systematic Review and MetaAnalysis. PLoS One. 2016;11:01633-65.

8. Khambaty M, Parikh RM. Cultural aspects of anxiety disorders in India. Dialogues Clin Neurosci. 2017; 19:117-26

9. Prakash S, Sharan P, Sood M. A study on phenomenology of Dhat syndrome in men in a general medical setting. Indian J Psychiatry. 2016;58:129-41

10. Ventriglio $A$, Ayonrinde $O$, Bhugra $D$. Relevance of culture-bound syndromes in the 21st century. Psychiatry Clin Neurosci. 2016;70:3-6.

11. Sameer M, Menon V, Chandrasekaran R. Is 'Pure' Dhat Syndrome a Stable Diagnostic Entity? A Naturalistic Long Term Follow Up Study from a Tertiary Care Centre. J Clin Diagn Res. 2015;9:1-3.

12. Faiad Y, Khoury B, Daouk S, Maj M, Keeley J, Gureje $\mathrm{O}$, et al. Frequency of use of the International Classification of Diseases ICD-10 diagnostic categories for mental and behavioural disorders across world regions. Epidemiol Psychiatr Sci. 2018;27:568-576. 
13. Chaturvedi S. Psychasthenic syndrome related to leucorrhea in Indian women. J Psychosom Obstet Gynaecol. 1988;8:67-72.

14. Chaturvedi SK, Chandra PS, Issac MK, Sudarshan CY. Somatization misattributed to non-pathological vaginal discharge. J Psychosom Res. 1993;37:57-59.

15. Bang R, Bang A. Perceptions of white vaginal discharge. In: Gittelsohn J, Bentley M, Pelto P, Nag M, Pachauri S, Harrison A, et al., editors. Listening to Women Talk about Their Health. New Delhi: HarAnand Publications; 1994. pp. 79-94.

16. Patel V, Oomman NM. Mental health matters too: Gynecological morbidity and depression in South Asia. Reprod Health Matters. 1999; 7:308.

17. Patel V, Pednekar S, Weiss H, Rodrigues M, Barros P, Nayak B, et al. Why do women complain of vaginal discharge? A population survey of infectious and pyschosocial risk factors in a South Asian community. Int JEpidemiol. 2005;34:853-62.

18. Patel V, Weiss HA, Kirkwood BR, Pednekar S, Nevrekar P, Gupte S, et al. Common genital complaints in women: The contribution of psychosocial and infectious factors in a population-based cohort study in Goa, India. Int J Epidemiol. 2006;35:1478-85.

19. Patel V, Andrew G, Pelto PJ. The psychological and social contexts of complaints of abnormal vaginal discharge: A study of illness narratives in India. J Psychosom Res. 2008;64:255-62
20. Patel V, Oomman N . Mental health matters too: Gynaecological symptoms and depression in South Asia, Reproductive Health Matters, 1999:7:14,30-8

21. Patel V, Pednekar S, Weiss H, Rodrigues M, Barros P, Nayak B, et al. Why do women complain of vaginal discharge? A population survey of infectious and pyschosocial risk factors in a South Asian community. Int JEpidemiol. 2005;34:853-62.

22. Agarwal P, Malik S, Padubidri V.A study of psychiatric morbidity in a gynaecology outpatient clinic. Indian Journal of Psychiatry 1990;32:57-63.

23. Bang R, Bang A. Listening to women talk about their reproductive health problems in the urban slums and rural areas of Baroda. Gittelsohn J, Bentley ME, Pelto PJ, eds. Listening to Women Talk about Their Health:Issues \& Evidente from India,New Delhi: Ford Foundation; 1996; p. 79-94.

24. Patel V, Pereira J, Coutinho L, Fernandes R, Fernandes J, Mann A. Poverty, psychological disorder and disability in primary care attenders in Goa, India. Br J Psychiatry. 1998;172:533-6

25. Orley J, Wing JK. Psychiatric disorder in two African villages. Arch Gen Psychiatry. 1979;36:513-20. 